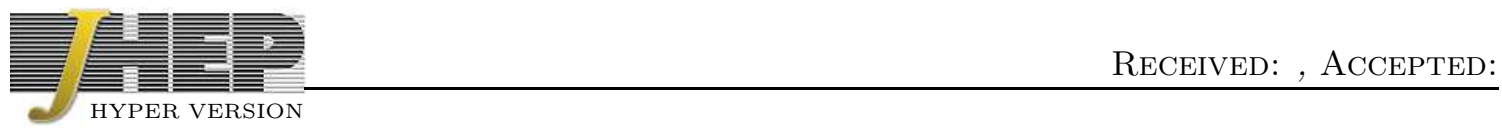

\title{
Proof of the entropy bound on dynamical horizons
}

\author{
Sijie Gao, \\ Department of Physics, \\ Beijing Normal University, \\ Beijing 100875, China \\ Email: sijie@bnu.edu.cn

\section{Xiaoning $\mathbf{W u}$ \\ Institute of Applied Mathematics, Academy of Mathematics and System Science, Chinese Academy of Sciences, Beijing, 100080,China. \\ Email: wuxn@amss.ac.cn}

ABSTRACT: The entropy bound conjecture concerning black hole dynamical horizons is proved. The conjecture states, if a dynamical horizon, $D_{H}$, is bounded by two surfaces with areas of $A_{B}$ and $A_{B^{\prime}}\left(A_{B^{\prime}}>A_{B}\right)$, then the entropy, $S_{D}$, that crosses $D_{H}$ must satisfy $S_{D} \leq \frac{1}{4}\left(A_{B^{\prime}}-A_{B}\right)$. We show that this conjecture is implied by the generalized Bousso bound. Consequently, the generalized second law holds for dynamical horizons. Finally, we show that the lightlike bousso bound and its spacelike counterpart can be unified as one bound.

KEYWORDS: entropy bounds, dynamical horizon. 


\section{Contents}

1. Introduction

2. Proof of the entropy bound related to a dynamical horizon 3

3. Gerneralizations of the proof 5

3.1 non-spherically symmetric case

3.2 The bound on a spacelike hypersurface 6

4. Conclusions 6

\section{Introduction}

Bekenstein [1] has conjectured that the entropy $S$ and energy $E$ of any thermodynamic system must obey

$$
S \leq 2 \pi E R
$$

where $R$ is defined as the circumferential radius. This bound is universal in the sense that it is supposed to hold in any matter system. The Bekenstein bound has been confirmed in wide classes of systems. However, as pointed by Bekenstein, the bound is valid for systems with finite size and limited self-gravity. Counterexamples can be easily found in systems undergoing gravitational collapse [2]. Another entropy bound is related to the holographic principle, which says that the entropy in a spherical volume satisfies

$$
S \leq \frac{A}{4}
$$

where $A$ is the area of the system. It was shown that this bound is violated for sufficiently large volumes [3]. Note that the entropy mentioned above is contained in a spacelike region. To find an entropy bound that has wider applications, Bousso investigated the entropy crossing a lightlike hypersurface and proposed a covariant entropy bound conjecture. Consider a spacelike 2 -surface $B$ with area $A_{B}$ in a spacetime satisfying the dominant energy condition. A null hypersurface $L$ is generated by null geodesics, which starts at $B$ and is orthogonal to $B$. Let $k^{a}$ be the tangent vector field of the geodesics and $\theta$ the expansion associated with $k^{a}$. Suppose that $\theta$ is nonpositive everywhere on $L$ and not terminated until a caustic is reached. Then the entropy, $S_{L}$, that crosses $L$ satisfies

$$
S_{L} \leq \frac{1}{4} A_{B} .
$$


Eq. (1.3) is the covariant entropy bound proposed by Bousso. This bound has passed the tests in various cases [2, 田, 5, 6]. A generalized Bousso bound allows $L$ to be terminated at any spacelike 2 -surface $B^{\prime}$ with area $A_{B^{\prime}}$ before coming to a caustic. Then Eq. (1.3) is modified as [1]

$$
S_{L} \leq \frac{1}{4}\left|A_{B^{\prime}}-A_{B}\right|
$$

This bound is obviously stronger than the original Bousso bound and its validity has been proved by assuming some physical conditions.

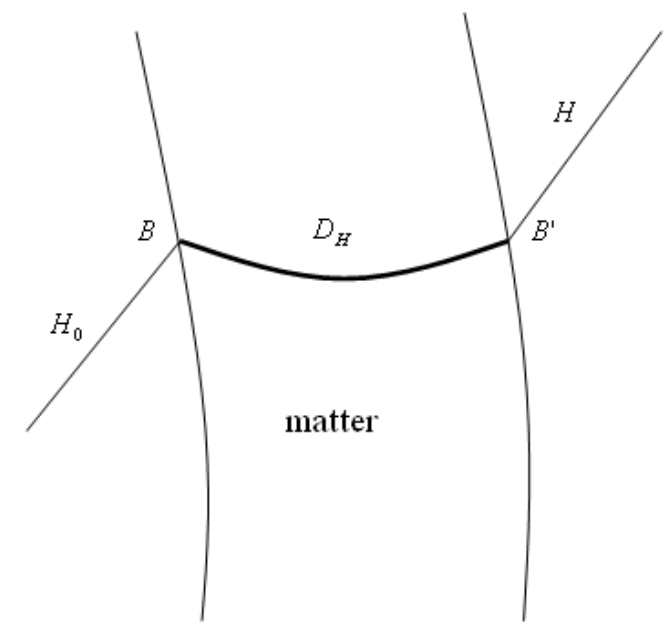

Figure 1: When matter collapses to an existing black hole, the dynamical horizon $D_{H}$ is formed, which connects the old horizon $H_{0}$ and the new horizon $H$.

Recently, an entropy bound related to a dynamical horizon has been discussed [7, 8]. A dynamical horizon is a spacelike hypersurface foliated by closed, marginally trapped 2-spheres, which describes an intermediate state when a black hole is formed[9]. Suppose that some matter is collapsing into an existing black hole. In Fig. $1, H_{0}$ represents the horizon of the existing black hole. A dynamical horizon $D_{H}$ begins to form at moment $B$. At moment $B^{\prime}$, the dynamical horizon finally evolves to an event horizon $H$ and a new black hole is formed. Since each point in Fig. 1 represents a closed 2-surface, we denote the areas at $B$ and $B^{\prime}$ by $A_{B}$ and $A_{B}^{\prime}$ respectively. Let $S_{D}$ be the entropy flux through the dynamical horizon. It has been shown in [9] that the area of the two-surface increases monotonically along $D_{H}$. It then follows that $A_{B^{\prime}}>A_{B}$, meaning that entropy of the black hole must increase and the second law of black hole mechanics holds [9]. However, this result is not enough to guarantee the generalized second law since the entropy of the matter has not been counted. Because the matter losses all its entropy after the formation of the new horizon $H$, the generalized second law is equivalent to the following bound

$$
S_{D} \leq \frac{1}{4}\left(A_{B^{\prime}}-A_{B}\right)
$$

This bound was given as a conjecture in [8] and its validity was tested only by a specific example. The purpose of our paper is to give a general proof for the conjecture. Apparently, 
the bounds (1.4) and (1.5) have the similar form. However, they are different in nature because the entropy in Eq. (1.4) crosses a null surface and the entropy in Eq. (1.5) is contained in a spacelike dynamical horizon. We have mentioned that the spacelike entropy bounds (1.1) and (1.2) can be easily violated for sufficiently large volumes where self-gravitation is strong. As a spacelike entropy bound, the validity of Eq. (1.5) is not obvious. On the other hand, the Bousso bound (1.3) and its generalized version (1.4) has passed all the tests so far in classical regimes. After investigating the relations between the generalized Bousso bound (1.4) and the spacelike bound (1.5), we find that the bound (1.5) is actually implied by the well-tested bound (1.4). Our proof in the next section will be based on the generalized Bousso bound as well as the properties of the dynamical horizon.

\section{Proof of the entropy bound related to a dynamical horizon}

In order to prove the bound (1.5) in a model as shown in Fig. 1, we make the following assumptions:

1. The generalized Bousso bound (1.4) holds.

2. The ordinary second law holds for the matter that crosses the horizon.

3. The spacetime is spherically symmetric.

4. The dynamical horizon is a future outer trapped horizon (FOTH) defined by Hayward 10.

The first assumption is essential in the following proof. The second assumption is quite natural. Assumption 3 simplifies the structure of the spacetime. The relevant dimension of spacetime is then reduced to two when only radial null geodesics are considered. We shall show later that this restriction can be released under certain conditions. We shall not use the original definition of dynamical horizon proposed by Ashtekar et.al. in [9]. Instead, we use a close but more restricted definition as stated in assumption 4. As proposed by Hayward [10], a FOTH is a spacelike hypersurface which is foliated by a family of closed two-surfaces, such that on each leaf

(i) the expansion of one future directed null normal $l^{a}$ vanishes, i.e., $\theta_{l}=0$.

(ii)the expansion of the other future directed null normal $n^{a}$ is negative, $\theta_{n}=0$.

(iii) $\mathcal{L}_{n} \theta_{l}<0$

The condition (iii) makes the FOTH differ from Ashtekar's dynamical horizon because it provides more information in a neighborhood of the horizon. As proven by Hayward, any spatial two-surface sufficiently close to a FOTH and in its past is not trapped. This property will be used in our following proof.

Since we are considering a dynamical horizon (FOTH) $D_{H}$, it follows by definition that $D_{H}$ is foliated by a family of closed 2-surfaces. Now consider a spacelike hypersurface, $D_{P}$, in the past of $D_{H}$ (see Fig. 2). A null geodesic starting from $B$ propagates backward to the past and bounces off at $B_{1}$ on $D_{P}$. If the light continues to propagate in this way, it will finally reach $B^{\prime}$ (or some point on $D_{H}$ close to $B^{\prime}$ ). We denote each point (representing a 2 -surface) by $B_{i}$ and its area by $A_{i}$. Since each section of the null line, denoted by $h_{i}$, represents a null hypersurface connecting two 2-surfaces, we may apply the generalized Bousso bound to each one of them. Note that the dynamical horizon is essentially the 


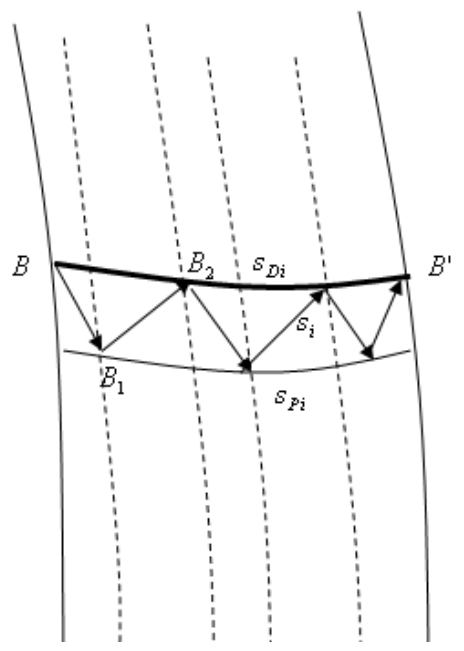

Figure 2: The short lines with arrows represent the ingoing and outgoing null geodesics. The dotted lines represent the comoving observers.

boundary of the trapped region and $D_{P}$ lies in its past. Therefore, according to Hayward's theorem, the foliations on $D_{P}$ are not trapped if $D_{P}$ is sufficiently close to $D_{H}$, which means that the expansion $\theta_{i}$ of each null generators of $h_{i}$ has a unique sign. For example, the null generators $B_{1} \rightarrow B$ and $B_{1} \rightarrow B_{2}$ have negative and positive expansions, respectively. Consequently, we have $A_{B}<A_{B_{1}}<A_{B_{2}} \ldots<A_{B^{\prime}}$. Denote the entropy crossing each small null surface by $s_{i}$. Then the bound (1.4) yields

$$
\begin{aligned}
s_{1} & \leq \frac{1}{4}\left(A_{B_{1}}-A_{B}\right) \\
s_{2} & \leq \frac{1}{4}\left(A_{B_{2}}-A_{B_{1}}\right) \\
& \cdots \cdots \\
s_{n} & \leq \frac{1}{4}\left(A_{B^{\prime}}-A_{B_{n-1}}\right)
\end{aligned}
$$

By adding all the inequalities above, we find

$$
\sum_{i=1}^{n} s_{i} \leq \frac{1}{4}\left(A_{B^{\prime}}-A_{B}\right) .
$$

Now we have obtained the right-hand side of the bound (1.5). But the left-hand side of (2.2) is the total entropy crossing all the small null surfaces, not the entropy crossing the dynamical horizon. To proceed, consider comoving observers passing through the connecting surfaces of the null geodesics (the dotted lines in Fig.2.). Denote the entropy that flows through an interval between two adjacent observers on $D_{P}$ by $s_{P i}$ and the corresponding one on $D_{H}$ by $s_{D i}$. By using our assumption 2, the ordinary second law, we have immediately

$$
s_{P i} \leq s_{i} \leq s_{D i}
$$


and by summation, we obtain

$$
S_{P} \leq \sum_{i=1}^{n} s_{i} \leq S_{D},
$$

where $S_{P}$ is the entropy crossing $D_{P}$. To complete the proof, we need to replace the left-hand side in Eq. (2.2) by $S_{D}$. Obviously, inequality (2.4) does not give the desired direction. This can be remedied by the following observation. Since our argument applies to any spacelike surface $D_{P}$ in the past of $D_{H}$, it applies to $D_{P}$ that approaches to $D_{H}$. When $D_{P}$ gets arbitrarily close to $D_{H}$, we have $S_{P} \sim S_{D}$. By virtue of Eq. (2.4), we have $\sum s_{i} \sim S_{D}$. Note that the right-hand side of Eq. (2.2) is a fixed value, which is independent of the limiting process. On the other hand, $S_{D}$ is a limit of $S_{P}$ and consequently a limit of $\sum s_{i}$. Therefore, if Eq. (2.2) holds for $\sum s_{i}$, it certainly holds for its limit $S_{D}$, as we desired to show.

\section{Gerneralizations of the proof}

Now we shall generalize our proof above from spherically symmetric case to non-spherically symmetric case and from a dynamical horizon to a general spacelike hypersurface.

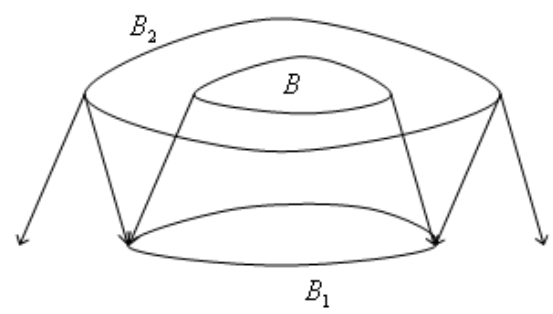

Figure 3: $B$ and $B_{2}$ are closed two-surfaces on $D_{H}$. $B_{1}$ is the intersection of the two light-sheets starting from $B$ and $B_{2}$.

\section{1 non-spherically symmetric case}

In the proof above, we see that spherical symmetry plays an important role. It guarantees that the light rays starting from $B$ will intersect $D_{P}$ and $D_{H}$ as two-spheres (i.e., $B_{1}, B_{2} \ldots$ in Fig. 2 are all two-spheres). Therefore, when $D_{P}$ is chosen close enough to $D_{H}$, the final cross-section of the light rays will always coincide with $B^{\prime}$ as shown in Fig.2. If the spherical symmetry is not imposed, the shape of the cross-sections will be out of control and the final cross-section will not coincide with $B^{\prime}$ in general. However, we can fix the problem by the following procedure. In the previous proof, the foliations on $D_{H}$ are the intersections of the light-sheets and $D_{H}$. In non-spherical cases, we may fix the foliations first, i.e., choosing $B, B_{2}, \ldots B^{\prime}$, on $D_{H}$. Then let the light rays propagate back to the past from each of these foliations inwardly and outwardly (see Fig[3). We assume that the intersections of the three-dimensional lightsheets are 2-dimensional spacelike surfaces, labeled by $B_{1}$, $B_{3}, \ldots$ Then our proof for spherical cases can be applied similarly. Therefore, the entropy 
bound (1.5) is generalized to non-spherically symmetrically dynamical horizons. Note that we have made additional assumption in the generalization, requiring the intersection of two lightsheets be a spacelike two-surface. This should not be a strong condition since we may choose the spacelike foliations on $D_{H}$ arbitrarily close.

\subsection{The bound on a spacelike hypersurface}

Our discussion above has been aimed at dynamical horizons. However, the relevant property we used is that there is a non-trapped region in a neighborhood of $D_{H}$ such that the inequalities (2.1) hold. It is not difficult to see that our proof applies to any spacelike hypersurface which lies outside a trapped or anti-trapped region. Thus, the lightlike Bousso bound (1.4) implies the spacelike entropy bound (1.5). Indeed, we may treat them as one unified entropy bound, which holds for lightlike hypersurface with negative expansion and spacelike hypersurface where no trapped or anti-trapped two-surface exists.

\section{Conclusions}

The entropy bound conjecture discussed in [7] and [8] has been proved based on the generalized Bousso bound and the ordinary second law. Therefore, the generalized second law is satisfied in the presence of a dynamical horizon. We also showed that the lightlike entropy bound and the spacelike entropy bound can be unified as one bound.

\section{Acknowledgements}

S.G. was supported in part by NSFC Grants 10605006, and the Scientific Research Foundation for the Returned Overseas Chinese Scholars, State Education Ministry. X.Wu was supported in part by NSFC Grants 10705048, 10605006, 10731080.

\section{References}

[1] J.D. Bekenstein, Phys. Rev. D 23, 287(1981).

[2] R. Bousso, J.High Energy Phys. 07 (1999) 004.

[3] W. Fishler and L. Susskind, hep-th/9806039

[4] E. Flanagan, D. Marolf and R. Wald, Phys. Rev. D 62, 084035 (2000).

[5] S. Gao and J.P.S.Lemos, J. High Energy Phys. 04(2004)017.

[6] S. Gao and J.P.S.Lemos, Phys. Rev. D 71, 084010 (2005).

[7] S. He and H. Zhang, J. High Energy Phys. 10(2007)077.

[8] S. He and H. Zhang, J. High Energy Phys. 12(2007)052.

[9] A. Ashtekar, B. Krishnan, Phys. Rev. D68, 104030 (2003).

[10] S.A.Hayward, Phys. Rev. D49, 6467 (1994). 